\title{
Éditorial:
}

\section{Des questions, politiquement hors de question, sur le régime de pension}

Traditionnellement, le rédacteur en chef de chacune des sections de $L a$ Revue canadienne du vieillissement organise, au terme de son mandat, un symposium spécial dans le cadre des rencontres annuelles éducatives et scientifiques de l'Association canadienne de gérontologie. Ce numéro de $L a$ Revue canadienne du vieillissement retrace les grandes lignes du symposium qui a eu lieu à Vancouver en octobre 1995 sous l'égide de Santé Canada, ${ }^{1}$ sous la rubrique $R e$-Writing Social Policy in an Aging Society. Les buts du symposium étaient triples: examiner la portée des changements proposés en matière de sécurité sociale, notamment la hausse de l'âge de la retraite; analyser l'effet de ces changements sur la santé et le bien-être des aînés; et interpréter ces enjeux dans un contexte international.

Au premier coup d'oeil, on peut être tenté de considérer les données du symposium comme de vieilles nouvelles. Après tout, les propositions sur les Prestations aux aînés ont été soumises, les consultations liées au Régime de pension du Canada sont terminées et les recommandations prévisibles vont suivre. La réduction de la dette et du déficit, leitmotiv connu, a permis que les compressions dans les régimes de pension soient correctement calculées et politiquement reconnues. Que dire de plus?

Voilà la véritable question. On pourrait élaborer longuement sur le sujet mais un silence pénétrant entoure le domaine gérontologique. Étouffés par les responsabilités fiscales, les Canadiens semblent croire qu'ils sont à la merci d'impératifs économiques. Même avec les meilleures intentions, les quelques rares personnes qui traitent des changements au régime de pension font presque infailliblement précéder leurs remarques d'un constat quant au caractère impérieux de la crise économique avant de couper la poire en deux dans leurs propositions pour contenir les coûts des programmes. En fait, ils ne vont pas au-delà des faux paramètres de la réduction de la dette prescrits par le ministère des Finances et par les marchés monétaires internationaux - en réduisant les coûts des programmes sociaux - alors que la plupart des économistes savent qu'il existe d'autres options (Freeman, 1995; Osberg \& Fortin, 1996). Nous en sommes venus à accepter passivement de ne pas remettre en question les politiques nationales fiscales et monétaires, de peur que les "marchés monétaires ne deviennent nerveux," ce qui a donné lieu à une vision presque apocalyptique de l'économie. Nous avons en quelque sorte oublié certains faits, par exemple, que ceux qui sont responsables des marchés monétaires tiennent à générer des profits pour leurs clients, souvent aux dépens du bien-être public (Van Audenrode, 1996) . 
Ce que nous élaborons ici ne constitue pas nécessairement une théorie du complot du type "on nous a eus," énoncée par un tenant de propagandes et d'analyses conservatrices (Freeman, 1995, p. 253) mais bien le danger qui nous guette de perdre confiance dans notre capacité de "critiquer, de rejeter le conformisme, la passivité et l'inévitable," contre toutes les attentes normales d'un citoyen vivant en démocratie (Saul, 1995, p. 36). Il en va de notre intérêt (la plupart d'entre nous allons prendre notre retraite), sans oublier le sens de notre démarche (les aînés), de remettre en question et critiquer les changements apportés à la sécurité sociale. Ces changements se fondent sur les exigences d'aujourd'hui qui vont être dépassées demain, selon toutes probabilités. Les articles de ce numéro soulèvent d'importantes questions sur la vision collective de notre système de sécurité sociale et sur le type d'économie morale qui se dessine pour l'avenir. L'économie morale repose sur des considérations liées à des échanges équitables dans notre société, à savoir quand et à qui accorder de l'aide et quand les privilégiés ont l'obligation de fournir de l'aide (Stone, 1984). À titre d'outil d'analyse, l'économie morale permet de clarifier comment nous passons d'une société essentiellement basée sur la valeur d'usage où l'on tente de maximiser les possibilités pour chacun d'avoir une vie décente, à une société où tout repose de plus en plus sur la valeur d'échange, laquelle s'appuie uniquement sur des indices économiques qui mesurent le bien public (Hendricks \& Leedham, 1991).

À partir d'un point de vue international, Anne-Marie Guillemard explique comment la topographie du cours de la vie a changé et comment les politiques sociales passent à côté du but visé. Le rafistolage pièce par pièce du système de pensions afin de contrer le chômage a entraîné des changements structurels difficilement réversibles. La retraite anticipée est pratique courante, les employés considérant les travailleurs plus vieux comme dépassés; ceux-ci ont adopté le même point de vue et planifient leur vie pour quitter le monde du travail tôt. On a procédé à la réforme des pensions indépendamment des autres aspects du système de sécurité sociale, ce qui a entraîné de nombreuses conséquences non voulues et a exclu le chevauchement de réformes socialement importantes au cours de la vie. La politique sociale fondée sur une vision tripartite de la vie (étude, travail, retraite) constitue un anachronisme puisque la vie se caractérise de plus en plus par des transitions multiples.

Susan McDaniel suit ce raisonnement et propose un nouveau concept comme ligne directrice pour cerner le changement dans la dynamique des individus au sein et en dehors de la famille, à l'intérieur du milieu de travail et en dehors de celui-ci et dans les programmes gouvernementaux qui traversent la vie d'un individu et des générations. Elle introduit le concept du «cycle des dépendances et des responsabilités de la vie» qui mesure les phases de transition, du soutien au partage, au plan individuel et entre les générations. Appliqué au microcosme, ce concept fait ressortir l'aspect privé et public en détaillant les dépendances et les responsabilités dans chacun des domaines. Au niveau macrocosmique, non seulement les différences 
entre les générations dans le cycle des dépendances et des responsabilités deviennent évidentes mais aussi, simultanément, des considérations quant au passé, au présent et au futur. Tout comme Guillemard, elle prône des changements dans les politiques sociales qui relieraient les politiques du marché du travail et celles du vieillissement et qui tiendraient compte des dépendances et des responsabilités qui traversent la vie et les générations.

Ces deux articles prédisent une transformation qui semble émerger autant ici qu'ailleurs: la féminisation du travail. Contrairement au sage point de vue qui prétendait que les carrières des femmes du baby boom ressembleraient plus à celle des hommes, un courant contraire semble se dessiner. La trajectoire de travail des hommes commence à ressembler plus à celle des femmes. Les hommes, aux deux extrémités de leur vie active, font partie de façon intermittente des forces de travail, en raison du taux élevé de chômage et des types d'emplois qui sont souvent non standards. Alors que plus de femmes que d'hommes travaillent à temps partiel, plus d'hommes que de femmes ont des emplois temporaires. Les revenus et les salaires moyens sont devenus stagnants et les hommes sont moins susceptibles de bénéficier d'une retraite professionnelle qu'il y a dix ans. La probabilité que les hommes retournent travailler après leur retraite semble s'accroître au fur et à mesure que la démarcation entre le travail et la retraite s'estompe. Cela fait de plus en plus partie de nos vies!

Comme le propose Guillemard, il est temps de réévaluer les transferts sociaux associés à la retraite. Les paiements de transferts ne devraient pas être faits en fonction de l'âge mais des besoins qui surgissent au cours de la vie. $\mathrm{McDaniel}$ conclut que des politiques réalistes seront formulées uniquement à la suite d'analyses de la vie de famille des gens, dans le contexte de l'histoire et dans celui d'une génération. Ces points de vue ont un potentiel considérable si la féminisation du travail se développe, mais même si tel n'est pas le cas, ces types de travail représentent néanmoins le sort des femmes qui sont exclues des politiques de pension. La question qui se pose alors est: pourquoi les concepteurs des politiques persistent-ils à élaborer des politiques qui se fondent sur un style de vie réglé sur le mode éducation, travail et retraite et fondées sur l'expérience d'un travailleur mâle de la société industrielle du XIXe siècle? Pourquoi les changements dans le cours de la vie sont-ils mis de côté? Pourquoi les politiques sociales sont-elles adaptées aux besoins du moment seulement? Pourquoi persistons-nous à construire des politiques sociales, une génération à la fois?

Frank Denton et Byron Spencer démontrent que l'apocalypse démographique ne proviendra pas du vieillissement des baby boomers; au lieu de cela, avec une soigneuse répartition des ressources entre les postes de dépenses et une planification à long terme, l'ensemble des hausses de coûts sociaux devrait être envisageable. Les auteurs examinent quatre mesures de rechange liées au «coût» d'une population vieillissante; le traditionnel ratio de dépendance; l'impact de la dépendance évalué différemment selon que les groupes sont âgés ou jeunes; les dépenses publiques prévues pour les personnes à charge plus âgées ou plus jeunes; et le pourcentage des 
dépenses publiques prévues par rapport au produit national brut. Ils estiment que nous devons adopter un point de vue à long terme pour concevoir le système de santé comme système intégré, et comme les soins de santé ne sont pas déterminés par les forces du marché, il incombe au gouvernement d'en assumer la direction. Comme les coûts de la sécurité sociale sont partiellement définis en fonction de la vieillesse, Denton et Spencer envisagent la possibilité de hausser l'âge d'admissibilité à la pension afin de refléter la prolongation de l'espérance de vie, sans oublier que l'âge de la retraite obligatoire à 65 ans devrait aussi être changé.

Robert Brown aborde les enjeux du vieillissement de la population d'un point de vue différent. La rhétorique de la démographie apocalyptique a causé tout un remous quant à la viabilité des régimes de pension fondés sur la répartition qui ont cours dans de nombreuses nations occidentales. Il soupèse soigneusement le pour et le contre du Régime de pension du Canada ( $R P C$ ) et du Régime de rentes du Québec (RRQ), régimes de répartition, et les compare au modèle à capitalisation anticipée comme celui du Chili. Il opte pour le modèle de répartition et le critique en détails pour illustrer comment le «super RRPS» parviendrait à assurer une sécurité de revenus aux aînés. Entre autres choses, le régime n'assurerait aucune distribution de revenus, caractéristique incompatible avec une économie morale basée sur la valeur d'usage dont les Canadiens semblent vouloir (Peters, 1995). Dans l'ensemble, les résultats de ses évaluations sont moins qu'encourageantes et il se demande pourquoi le "super RRPS" compte encore des défenseurs. Si Brown s'interroge, tous devraient le faire, particulièrement à la lumière du récent fiasco de Bre-X. Espérer assurer son revenu de retraite en s'adressant à des gestionnaires financiers professionnels qui investiront dans des actifs du secteur privé est pour le moins inquiétant en cette période incertaine. Enfin, Brown se prononce en faveur de la hausse de l'âge de la retraite, non pour réduire les coûts du régime de pension de l'état en soi, mais pour résoudre ce qu'il appelle le dilemme des transferts liés à la santé, c'est-à-dire des transferts servant à l'éducation et aux soins de santé des jeunes qui sont moindres que les transferts consacrés aux soins de santé et aux rentes des personnes âgées, facteur d'instabilité économique.

Pour de nombreuses personnes, la hausse de l'âge de la retraite pourrait être positive - les travailleurs qui sont forcés de prendre leur retraite avant le temps, les travailleurs qui se sentent inutiles, les employeurs qui maintiendraient dans leurs fonctions des travailleurs expérimentés et des contribuables puisque les coûts des régimes de pension gouvernementaux seraient amoindris et les contributions au RPC et au RRQ seraient réduites. L'idée n'est pas mauvaise dans la mesure où les plus vulnérables, soit les malades et les pauvres, peuvent bénéficier de protection par le biais de dispositions spéciales. Alors pourquoi les gouvernements ne discutent-ils pas de cette possibilité? Ken Battle, qui prétend que hausser l'âge de le retraite constitue une mesure régressive, indique dans son article «An Information Paper for Consultations on the Canada Pension Plan» (Gou- 
vernements fédéral et provincial, 1996) que la réforme du RPC et du RRQ n'offre pas une multitude d'options, car certaines ont déjà été exclues. Le vrai débat sur les solutions de rechange se tiendra derrière des portes closes entre les gouvernements fédéral et provincial. Il serait intéressant de savoir quelles options ont été éliminées, qui les a éliminées et sur quelles bases elles l'ont été et, qui plus est, pourquoi le public est exclu du débat.

La recherche de Denton et Spencer sur la redistribution des dépenses de programmes et l'analyse de Brown sur les projets de capitalisation soulèvent la plus impopulaire de toutes les questions - la réduction des dépenses fiscales allouées pour les REER et les RER. Nous semblons oublier de façon systématique que l'argent non encaisse à partir des impôts constitue une dépense gouvernementale tout comme l'argent dépensée dans les programmes sociaux. Environ 15 milliards de dollars (Shillington, 1996) pourraient être épargnés en éliminant ces dépenses qui sont vraiment une forme d'assistance sociale déguisée pour les mieux nantis, aux dires même de Battle. Le point de vue selon lequel nous avons besoin de capital d'investissement revêt moins d'importance étant donné que nous disposons d'un surplus à l'heure actuelle (Brown, dans ce numéro).

L'article de Battle retrace l'histoire des changements dans les régimes de pension au Canada, laquelle culmine par une analyse critique des nouvelles Prestations aux aînés de même que sur les modifications prévues au $R P C$ et au RRQ. Son examen comporte quelques remarques déconcertantes. Au cours du "grand débat sur les pensions" qui a eu lieu au début des années 1980, il y a eu des discussions substantielles avec toutes les parties intéressées, particulièrement le grand public. Toutefois, comme le mentionne Battle, il y a eu beaucoup de discussions et peu d'action. Aujourd'hui, il y a beaucoup d'actions et peu de discussion, préoccupation centrale de notre éditorial. Battle lui-même fait ressortir que sa proposition de réforme des pensions qui constitue le fondement des Prestations aux aînés a suscité peu de commentaires, si ce n'est parmi un petit groupe d'analystes de politiques sociales et au sein de quelques groupes nationalistes (Conseil consultatif national sur le troisième âge, 1996; Conseil national du bien-être, 1996).

Selon lui, une partie du problème concerne la stratégie de mise en oeuvre employée - les Prestations aux aînés ont été présentées comme un "fait accompli» et calculé pour dompter l'opposition en maintenant les droits acquis des personnes qui sont actuellement âgées.

Il a souligné certains des changements anticipés dans l'article de Brown qui vraisemblablement feront surface dans les changements du RPC et du $R R Q$, lesquels sont relativement inappropriés - capitalisation partielle, hausse des taux de contribution et réductions des prestations. S'il subsiste un doute quelconque quant aux effets dévastateurs qu'entraîneront plusieurs de ces changements, mon article sur les veuves retraitées le dissipera rapidement. On sait pertinemment que la pauvreté chez les femmes est bien souvent reliée à l'absence ou la perte du mari. L'analyse démontre que 49 pour 100 des veuves retraitées vivent sous le seuil de la pauvreté et ont un 
revenu plus bas que les femmes mariées, séparées, divorcées ou célibataires. Les résultats ne peuvent être écartés comme effet de cohorte avec le point de vue habituel que les femmes recevront éventuellement leur propre pension. Aujourd'hui, moins de la moitié des femmes ont leur propre pension professionnelle et le travail intermittent et à temps partiel des emplois peu rémunérés laisse entrevoir peu de signes de changements.

J'aborde dans le sens de Battle sur la controverse qui entoure de nombreux aspects des Prestations aux aînés mais peu d'experts du vieillissement semblent prendre part au débat. Quoiqu'il en soit, chacun des articles de ce numéro soulève indirectement de sérieux points faibles du régime des Prestations aux aînés. D'abord, les prestations constituent un changement au programme social quand on aurait peut-être pu envisager d'autres formes de réduction de coûts (Battle). Ne serait-ce que sous l'aspect mathématique du programme, a-t-on seulement envisagé d'autres catégories de dépenses (Denton \& Spencer)? Le nouveau régime ne reflète pas une vie en évolution et ses multiples transitions à tous moments (Guillemard). Soixante-cinq ans demeure l'âge d'admissibilité à la retraite sans plus de flexibilité dans le programme; un retour au travail suite à une retraite, même s'il s'agit d'un retour à temps partiel, entraînera des pénalités. Si le monde du travail continue d'aller dans le sens d'emplois plutôt non standards, il est fort probable que les générations futures dépendront très largement des Prestations aux aînés. Les prestations sont un attrape-nigauds, un exemple de mesure hésitante qui ne ramène pas le revenu des personnes âgées pauvres au-dessus du seuil de la pauvreté établi par Statistique Canada (McDonald).

Le soutien et le partage au sein des générations actuelles et futures sont des éléments exclus explicitement du régime des Prestations aux aînés, de même que l'interrelation entre le travail et la famille (McDaniel, Brown). À cet égard, le régime est subordonné au revenu et représente ce que Margrit Richler appelle le familism, un concept qui fait partie intégrante du syndrome sexiste. Les Prestations aux aînés place la famille au rang de "la plus petite unité d'analyse alors que ce sont, en fait, les individus à l'intérieur des familles qui s'engagent dans certaines actions, ont certaines expériences, souffrent ou profitent de coûts ou de prestations particulières" (Richler, 1997, p. 90). Tous les gérontologues savent que le rôle de soignante adopté par les femmes à différents moments de leur vie affecte négativement leur carrière, leur pension et, en bout de ligne, leur revenu de retraite. Suivant les Prestations aux aînés, leur récompense pour accomplir une fonction sociétale essentielle se résume à une rente inexistante pour ellesmêmes, et/ou très peu de rente, car elle sera basée sur le revenu de leur mari (habituellement le revenu le plus élevé de la famille et celui devant être utilisé pour le calcul des prestations). Il est de plus proposé plus loin que le montant des prestations soit divisé également en deux chèques émis séparément au mari et à la femme, soit une forme de reconnaissance monétaire quant à la contribution des femmes dans la société. On peut se demander pourquoi les femmes ne reçoivent pas une reconnaissance directe 
de leur contribution substantielle, non seulement à la famille, mais aussi à l'économie canadienne, tout comme les hommes? Cela soulève une autre question intéressante, à savoir pourquoi les plafonds des REER, de même que leurs prestations, sont-ils applicables aux revenus individuels et aux contributions, et non pas aux revenus de la famille (Shillington, 1995)? Pourquoi les prestations devraient-elles être différentes?

Il y a, bien sûr, de nombreux autres point de vue, parfois opposés, à découvrir dans ces articles, mais il n'en demeure pas moins que les questions doivent être posées et les réponses doivent être analysées de façon critique. Nous réclamons sincèrement un autre "grand débat sur les pensions" qui réunisse non seulement des analystes politiques mais aussi des gérontologues et des experts des milieux financiers. Alors, pour terminer avec la plus politique des questions hors de question, nous devons nous demander «Pourquoi ne sommes-nous pas mis à contribution?»

\section{Note}

1 Je tiens à remercier Louise Plouffe pour avoir co-présidé le symposium Re-Writing Social Policy for an Aging Society, de même que pour son appui indéfectible à l'Association canadienne de gérontologie.

\section{Références}

Eichler, M. (1997). Family shifts: Families, policies, and gender equality. Toronto: Oxford University Press.

Freeman, R.B. (1995). W(h)ither the welfare state in an epoch of rising inequality? Dans K. Banting \& C.M. Beach (Éds.), Labour market polarization and social policy reform. Kingston: School of Social Policy Studies, Queen's University.

Gouvernements fédéral et provincial. (1996). An information paper for consultations on the Canada Pension Plan. Ottawa

Hendricks, J., \& Leedham, C.A. (1991). Political economy of aging. Dans M. Minkler \& C.L. Estes (Éds.), Critical perspectives on aging: The political and moral economy of growing old. Amityville, NY: Baywood Pub. Co., Inc.

National Advisory Council on Aging. (1996). The National Advisory Council on Aging brief on the five principles for federal pension reform.

National Council of Welfare. (1996). A guide to the proposed seniors benefit. Ottawa: Minister of Supply and Services Canada.

Osberg, L., \& Fortin, P. (Éds.). (1996). Unnecessary debts. Toronto: James Lorimer and $\mathrm{Co}$.

Peters, S. (1995). Exploring Canadian values: A synthesis report. Ottawa: Canadian Policy Research Networks, Inc.

Saul, J.R. (1995). The unconcious civilization. Concord, ON: House of Anansi Press Ltd.

Shillington, R. (1995). The targetting paradox. Insight. Ottawa: Canadian Council on Social Development.

Shillington, R. (1996). The tax system and social policy reform. Dans J. Pulkingham \& G. Ternowetsky (Éds.), Remaking Canadian social policy: Social security in the late 1990s (pp. 100-111). Halifax: Fernwood Pub.

Stone, D.A. (1984). The disabled state. Philadelphia: Temple University.

Van Audenrode, M. (1996). Some myths about monetary policy. Dans L. Osberg \& P. Fortin (Éds.), Unnecessary debts. Toronto: James Lorimer and Co.

Lynn McDonald 